\title{
AN ONLINE PATH PLANNING METHOD BASED ON HYBRID QUANTUM ANT COLONY OPTIMIZATION FOR AUV
}

\author{
Changjian Lin, ${ }^{*}$ Hongjian Wang, ${ }^{*}$ Jianya Yuan,* and Mingyu Fu*
}

\begin{abstract}
Path planning is one of the important autonomy abilities for autonomous underwater vehicle (AUV), whose main purpose is to plan an optimized and safety path autonomously during long-range navigation in an unknown environment. This paper proposes two path planners based on quantum ant colony optimization (QACO) and hybrid QACO for AUV in real time based on a sensor detection window. When AUV detects unknown static obstacles, the online path planners are scheduled to plan out a new path to avoid obstacles in the optimization window. To limit the yaw angle, a nonlinear fitness function is defined. In the hybrid QACO, an adaptive quantum gate and improved rules of pheromone updating are proposed according to the movement characteristic of AUV in the process of obstacle avoidance. A local search method is combined with QACO to improve the quality of the path planned by QACO and obtain a smoother path. Finally, the simulation experiments compare the performance of the proposed path planning methods with ant colony optimization.
\end{abstract}

\section{Key Words}

Autonomous underwater vehicle (AUV), online path planning, hybrid quantum ant colony optimization, local search method, adaptive quantum gate

\section{Introduction}

Autonomous underwater vehicle (AUV) has been applied to scientific research (including oceanography, geomorphology, physiognomy, etc.), military field, environmental protection, marine engineering and some other commercial areas widely. More and more intensity for the exploitation of AUV is needed with more extensive application. Autonomous planning is one of the kernel technologies for AUV and is widely studied by many researchers. The path planning is further divided into global path planning

\footnotetext{
* College of Automation, Harbin Engineering University, Harbin, People's Republic of China; e-mail: $\{18243088393$, cctime99\}@ 163.com, 2414450031@qq.com, fumingyu@hrbeu.edu.cn Corresponding author: Hongjian Wang Recommended by Dr. Sivalinga Ponnambalam (DOI: 10.2316/Journal.206.2018.4.206-5337)
}

and local path planning. Global path planning assumes that the environment is completely known before the robot begins its traverse. Its purpose is to find a collision-free path from the source position to the destination position according to some optimization criteria [1]. But local path planning enables a robot to plan its path as it is moving in the environment, which means that the robot will be able to generate new paths to response the changes of environment [2].

In recent years, many effective algorithms for global path planning have been proposed. Zhang et al. constructed heuristic information by the concept of artificial potential field to improve ACO for AUV path planning [3]. The improved ACO can maintain a safe distance between obstacles and AUV. But the yaw angles of paths planned by the improved ACO are too large and do not conform the yaw characteristics of AUV. Liu et al. improved ACO through combined pheromone diffusion with geometric local optimization in the process of searching the globally optimal path for mobile robots. The proposed algorithm solved the problems of convergence speed in traditional ACO [4]. Yao et al. proposed a heterogeneous feature ant colony optimization (HFACO) algorithm in the robot path planning problem. In HFACO, two kinds of ants that ant A finds the path by searching randomly and extensively and leaves pheromone for ant B to exploit and optimize the path are defined [5]. The HFACO is efficient to shorten the execution time. But it is easy to run into local optimum when the map scale gets larger. The nonuniformity initial distribution of pheromone and heuristic strategy with direction information is presented in ACO by Zhao et al. for path planning of the omnidirectional mobile vehicle. In addition, the coverage and updating strategy is defined in the process of pheromone update to avoid repeated search. These improvements enhance the time efficiency and the simplicity of the algorithm and reduce the search space of ant colony algorithm [6]. Cekmez et al. implemented UAV path planning by using multi-colony $\mathrm{ACO}$ where $\mathrm{N}$ ACO works for the same problem set distributively and shares their local colony knowledge among the other colonies at specific intervals [7]. The multi-colony ACO can reduce the probability of trapping in local optimum, but the algorithm may have a long period of stagnation in the process 
of convergence. To enhance the global searchability and convergence speed of ACO, Cao proposed an improved ACO and applied it to solve the robot global path planning problem. In the improved ACO, pheromone quantity is reinforced in some short paths of each cycle, and the pheromone evaporation rate is adjusted dynamically with the change of iterations [8]. Deng et al. combined artificial immune network algorithm (AINA) and position tracking control method to solve the problem of multirobot formation path planning [9], in which the steering direction of the follower robot is calculated with AINA. And the simulation results demonstrated the performance of the algorithm in a simple environment. Li et al. proposed a hybrid path planning algorithm for unmanned aerial/ground vehicle cooperative systems. The hybrid algorithm consists of a genetic algorithm used for global path planning and the local rolling optimization that is used to constantly optimize the global path [10]. Due to the limitations of the environment model, the global path planned by the hybrid path planning algorithm is not smooth enough and the optimal path may be ignored. Fan et al. improved the classical Dijkstra's algorithm by limiting the searching area of algorithm and improve the storage structure of data based on the municipal transportation cyberspace distribution characteristic [11]. Dolgov et al. use Voronoi diagrams and potential fields together to overcome the shortcomings in each planner for mobile robots [12].

Some research has addressed online path planning for the unmanned system. Nieto et al. proposed an online path planning method based on rapidly exploring random trees for mobile robot [13]. The path planner can generate a collision-free path, but the optimal path may be ignored sometimes. Zhang et al. presented a Q-learning method for path planning in a dynamic environment. In the same manner, the robot is able to use the past experience for improving its performance in avoiding obstacles [14]. A machine-learning-based algorithm for detecting a moving object was proposed by Zhu et al. [15]. Chen et al. proposed an immune genetic algorithm with elitism (IGAE) to optimal path planning for mobile robots [16], in which a new antibody similarity was defined to reduce calculation amount and improve convergence speed. But the quality of solution was not improved by the proposed IGAE. Yang et al. proposed a real-time finite angle $\mathrm{A}^{*}$ to plan path in real time for unmanned surface vehicle (USV) [17]. Yu et al. developed a motion planning algorithm based on the hybrid search fast marching method for AUV, which can find continuous smooth paths within time constraints [18]. Zhu et al. proposed an enhanced artificial potential field method to deal with the obstacle avoidance for AUV [19]. Gong et al. proposed a local optimal strategy based on particle swarm optimization to handle the uncertain information for robot path planning [20]. In the proposed method, fewer path segments result in better real-time performance but poor optimal effect. Cheng et al. proposed a dynamic path planning strategy of adaptive chaotic particle swarm optimization algorithm for UAV [21]. The article has proved that the improved PSO is more effective to plan a track for UAV than standard PSO and immune PSO. Huang et al. proposed a fuzzy logic system for the obstacle avoidance of mobile robot [22]. Sfeir et al. dealt with the navigation of a mobile robot in an unknown environment based on an improved artificial potential field method. In the improved method, a new form of repelling potential is proposed to reduce oscillations and to avoid conflicts when the target is close to obstacles [23]. The trajectory smoothness and length was improved by the new method, but it cannot improve the iterative searchability of the global optimal solution. Some improved ant colony system algorithms which implemented real-time path planning of mobile robot has been proposed [24], [25]. These algorithms may take some time to converge to an optimal solution, which will affect the safety of robots. And the performance of these algorithms is bad when avoiding some complex obstacles.

Different from ACO, Q-bit and quantum rotation gate are adopted in QACO to represent and update the pheromone, respectively. Wang et al. defined a new update strategy of rotation angle, because the traditional strategy used in quantum-inspired evolutionary algorithm (QEA) is improper for QACO [26]. They also proved that $\mathrm{QACO}$ is a competitive optimization algorithm to solve discrete binary optimization problems compared with QEA and DBPSO. Li et al. combined QEA with ACO to solve travelling salesman problem (TSP) which improved the diversity and global search capacity of ACO [27]. For the shortcoming of ACO which was only suitable for the discrete optimization and accelerate the convergence, $\mathrm{Li}$ et al. proposed a suitable QACO for continuous optimization. In the new method, the information of ants were directly encoded by the phase of qubits. And a Pauli-Z gates was adopted to increase the diversity of ants [28]. To improve the efficiency and performance of ACO in TSP problems, Ma et al. proposed a new QACO, in which the models of probability selection and pheromone were redefined [29].

Compared with mobile robot and USVs, AUVs have some limitations in dexterity and perception. Focusing on the characteristics of AUV, this paper proposes two online path planners used in an unknown environment based on QACO and hybrid QACO. The hybrid QACO combines QACO with a local search method to solve the shortcomings exist in QACO and ACO, such as slow convergence, local minimal and poor robustness. The simulation results show that the hybrid QACO is a simple quick and efficient algorithm. It can dynamically plan an obstaclefree path for AUV quickly even in a complex unknown environment.

The structure of this paper is as follows. The simulation model and coordinate transformation of sensor are introduced in Section 2. Section 3 details the hybrid QACO used to plan path for AUV. Then, in Section 4, the process of online path planning based on hybrid QACO is narrated. Section 5 compares the performance of hybrid QACO, $\mathrm{QACO}$ and ACO in a two-dimensional environment. And the inline path planning ability of hybrid QACO in a threedimensional environment is demonstrated in Section 5 . Finally, Section 6 concludes the main works of this paper. 


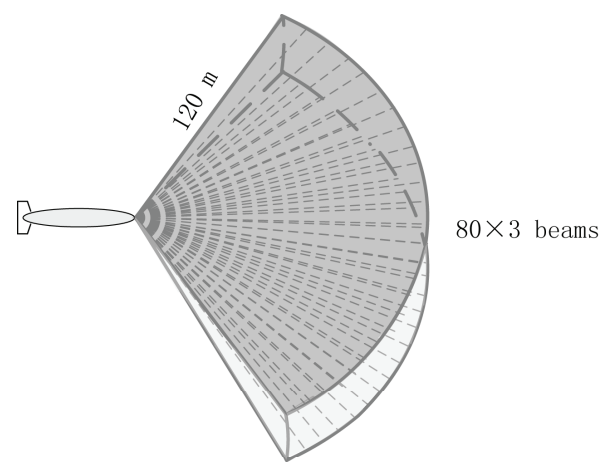

Figure 1. The main parameters of multibeam FLS.

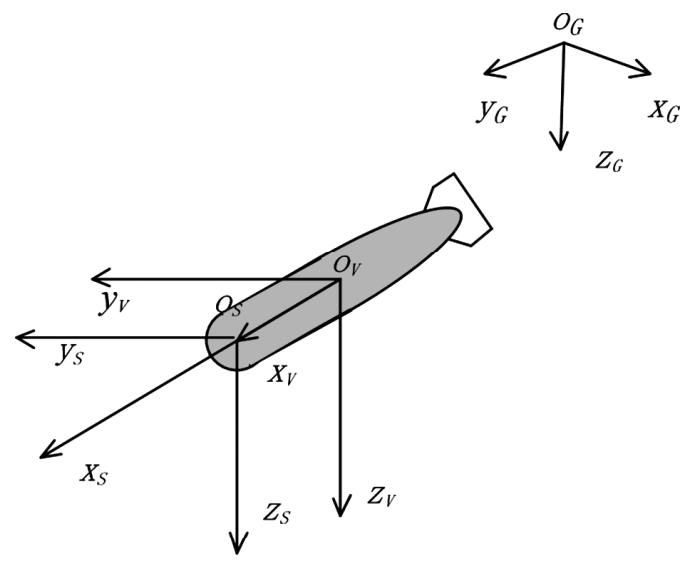

Figure 2. The positional relationship between the three coordinate systems.

\section{Simulation Model of Sensor}

\subsection{The Main Parameters of Multibeam Forward- Looking Sonar}

Figure 1 shows the main parameters of the multibeam forward-looking sonar (FLS) [30]. Sonar horizontal open angle is $120^{\circ}$, the vertical open angle is $17^{\circ}$, the number of horizontal beams is 80 , the number of vertical beams is 3 and the scan radius of multibeam FLS is $120 \mathrm{~m}$. Sonar can detect obstacles in its vision, calculates the coordinates of the intersection of the surface of the obstacle and 240 beam rays and calculates the distance of sonar head and the intersection.

\subsection{The Information Processing of Multibeam FLS in Three-Dimensional Environment}

The basic idea of identifying obstacles surface feature point is as follows. According to the distance between 240 beams and the surface of the obstacle to calculate the coordinates of obstacle surface feature point in the hull coordinate system, transform the coordinates of obstacle surface feature points from the hull coordinate system to the earth coordinates. The positional relationship between the three coordinate systems is shown in Fig. 2 [31].

In Fig. 2, $x_{G} y_{G} z_{G}$ is the north-east-down coordinate system, $x_{V} y_{V} z_{V}$ is the coordinate system of AUV and $x_{S} y_{S} z_{S}$ shows the FLS coordinate system.

According to the principle of sound waves, the intersection of the beam and obstacles must be the obstacle surface feature points. Suppose beam3-Gram $(i n \operatorname{dex}(i, j), l)$, where $i, j$ are the angle of the FLS and the obstacle, $l$ is the distance between FLS and the obstacle. The point $p$ corresponding to the coordinates of the obstacle surface feature points in the FLS coordinate system is $\left(x_{S}^{p}, y_{S}^{p}, z_{S}^{p}\right)$ :

$$
\begin{aligned}
& x_{S}^{p}=l \cdot \cos ((i+0.5) \cdot \beta / 3-\beta / 2) \cdot \cos ((j+0.5) \cdot \alpha-\alpha / 2) \\
& y_{S}^{p}=l \cdot \cos ((i+0.5) \cdot \beta / 3-\beta / 2) \cdot \sin ((j+0.5) \cdot \alpha-\alpha / 2) \\
& z_{S}^{p}=l \cdot \sin ((i+0.5) \cdot \beta / 3-\beta / 2)
\end{aligned}
$$

Then, the point $p$ can be expressed as $\left(x_{V}^{p}, y_{V}^{p}, z_{V}^{p}\right)$ in the AUV coordinate system:

$$
\begin{aligned}
x_{V}^{p}= & l_{\text {as }}+l \cdot \cos ((i+0.5) \cdot \beta / 3-\beta / 2) \\
& \cdot \cos ((j+0.5) \cdot \alpha-\alpha / 2) \\
y_{V}^{p}= & 0+l \cdot \cos ((i+0.5) \cdot \beta / 3-\beta / 2) \\
& \cdot \sin ((j+0.5) \cdot \alpha-\alpha / 2) \\
z_{V}^{p}= & 0+l \cdot \sin ((i+0.5) \cdot \beta / 3-\beta / 2)
\end{aligned}
$$

where $l_{a s}$ is the distance between FLS position and centroid of AUV, $\alpha$ is the horizontal open angle of FLS and $\beta$ is the vertical open angle of FLS.

According to the theory of ship moving model building, the coordinates of obstacle surface feature points in the earth coordinate system can be expressed as follows:

$$
\left[\begin{array}{c}
x_{G}^{p} \\
y_{G}^{p} \\
z_{G}^{p}
\end{array}\right]=\mathbf{S} \cdot\left[\begin{array}{c}
x_{V}^{p} \\
x_{V}^{p} \\
x_{V}^{p}
\end{array}\right]+\left[\begin{array}{c}
x_{G}^{V} \\
y_{G}^{V} \\
z_{G}^{V}
\end{array}\right]
$$

$$
\mathbf{S}=\left[\begin{array}{ccc}
\cos \psi \cos \theta & \cos \psi \sin \theta \sin \varphi-\sin \psi \cos \varphi & \cos \psi \sin \theta \cos \varphi-\sin \psi \sin \varphi \\
\sin \psi \cos \theta & \cos \psi \sin \theta \sin \varphi+\cos \psi \cos \varphi & \sin \psi \sin \theta \cos \varphi-\cos \psi \sin \varphi \\
-\sin \theta & \cos \theta \sin \varphi & \cos \theta \cos \varphi
\end{array}\right]
$$


where $\left(x_{G}^{V}, y_{G}^{V}, z_{G}^{V}\right)$ is AUV's position in the earth coordinate system, $(\varphi, \theta, \psi)$ is AUV's attitude angle and $\left(x_{G}^{p}, y_{G}^{p}, z_{G}^{p}\right)$ is the earth coordinate of point $p$. The coordinate of obstacle surface feature point $p$ in the AUV coordinate system can be expressed as $\left(x_{V}^{p}, y_{V}^{p}, z_{V}^{p}\right)$.

\subsection{Simulation Model of Multibeam FLS in Two- Dimensional Environment}

For being convenient to study, a two-dimensional sonar simulation model is established. For a two-dimensional sonar model, the sonar open angle is $120^{\circ}$, it has 80 beams, and the scan radius is $120 \mathrm{~m}$. The simulate sonar can detect obstacles in its vision, calculates the coordinates of the intersection of the surface of the obstacle and 80 beam rays and calculates the distance of sonar head and the intersection.

Suppose the beam $\operatorname{bigram}(\operatorname{index}(j), l)$, the point corresponding to the coordinates of the obstacle surface feature points $p$ in the FLS coordinate system is $\left(x_{S}^{p}, y_{S}^{p}\right)$, then

$$
\begin{aligned}
& x_{S}^{p}=l \cdot \cos (j \cdot \alpha / 80-\alpha / 2) \\
& y_{S}^{p}=l \cdot \sin (j \cdot \alpha / 80-\alpha / 2)
\end{aligned}
$$

The point $p$ can be expressed as $\left(x_{V}^{p}, y_{V}^{p}\right)$ in the AUV coordinate system, and

$$
\begin{aligned}
& x_{V}^{p}=l_{\text {as }}+l \cdot \cos (j \cdot \alpha / 80-\alpha / 2) \\
& y_{V}^{p}=0+l \cdot \sin (j \cdot \alpha / 80-\alpha / 2)
\end{aligned}
$$

where $l_{a s}$ is the distance between multibeam FLS position and centroid of AUV, $\alpha$ is the horizontal open angle of sonar.

Suppose AUV's position is $\left(x_{G}^{V}, y_{G}^{V}\right)$ in the earth coordinate system, the heading is $\theta$, the coordinates of point $p$ is $\left(x_{G}^{p}, y_{G}^{p}\right)$ in the earth coordinate system. And the coordinate of obstacle surface feature point $p$ in the AUV coordinate system can be expressed as $\left(x_{V}^{p}, y_{V}^{p}\right)$, then

$$
\begin{gathered}
{\left[\begin{array}{c}
x_{G}^{p} \\
y_{G}^{p}
\end{array}\right]=\mathbf{S} \cdot\left[\begin{array}{l}
x_{V}^{p} \\
x_{V}^{p}
\end{array}\right]+\left[\begin{array}{l}
x_{G}^{V} \\
y_{G}^{V}
\end{array}\right]} \\
\mathbf{S}=\left[\begin{array}{ll}
\cos \theta & -\sin \theta \\
\sin \theta & \cos \theta
\end{array}\right]
\end{gathered}
$$

\section{Hybrid Quantum Ant Colony Optimization}

In the hybrid QACO, the improved rules of pheromone updating and a local search method are proposed. When the improved algorithm is called, according to the selection probability, the algorithm chooses the next path point based on the pheromone matrix and environment information, and then a local search method is called to optimize or amend the solution. Each ant in hybrid QACO carries a set of quantum bits that represent the current position information of the ant, which doubles the search space when the number of ants is the same. Hybrid QACO uses a quantum rotation operation to increase the diversity of population positions and avoid premature convergence, which overcomes the defect that the ant colony algorithm is easy to fall into the local optimal because of the loss of population diversity in the search space. In the process of algorithm optimization, the quantum rotation gate is used to update the pheromone carried by ants to accelerate the convergence speed. Finally, a local search method is used to optimize the feasible solution or amend the infeasible solution.

\subsection{Encoding Method}

The mathematical description is expressed by a set of quantum bits. The nodes position of the quantum ants is represented by the corresponding probability of these quantum bits. Quantum pheromone is encoded with quantum bits. Suppose that there are $n$ ants in the colony, and quantum pheromone on the quantum ant path is expressed by $m$ quantum bits, that is, the dimension of hybrid QACO is $m$. This paper uses binary encoding, and then a group of quantum bits ant colony carried is described as follows:

$$
P=\left(p_{1}, p_{2}, \ldots, p_{j}, \ldots, p_{n}\right), \quad p_{j}=\left[\begin{array}{c|c|c|c}
\alpha_{j 1} & \alpha_{j 2} & \cdots & \alpha_{j m} \\
\beta_{j 1} & \beta_{j 2} & \cdots & \beta_{j m}
\end{array}\right]
$$

where $\left|\alpha_{j i}\right|^{2}+\left|\beta_{j i}\right|^{2}=1, \quad i=1,2, \ldots, m, \quad j=1,2, \ldots, n$, $|\alpha|^{2}$ and $|\beta|^{2}$ represent pheromone of the two paths that quantum ant to be selected, respectively. For each ant in each dimension, $\alpha$ and $\beta$ are initialized to $1 / \sqrt{2}$. It is easy to know that the $j$ th quantum ant has $2^{m}$ quantum states. Therefore, the search space of the algorithm is greatly increased, and the search speed of the algorithm is improved accordingly.

\subsection{The Adaptive Quantum Rotation Gate}

Quantum bits are updated according to the operation of rotation gate which makes the ant colony develop to the best individual:

$$
\left[\begin{array}{c}
\alpha_{j i}^{\prime} \\
\beta_{j i}^{\prime}
\end{array}\right]=\mathbf{R}\left(\theta_{i}\right)\left[\begin{array}{c}
\alpha_{j i} \\
\beta_{j i}
\end{array}\right]=\left[\begin{array}{cc}
\cos \theta_{i} & -\sin \theta_{i} \\
\sin \theta_{i} & \cos \theta_{i}
\end{array}\right]\left[\begin{array}{c}
\alpha_{j i} \\
\beta_{j i}
\end{array}\right]
$$

where $\theta_{i}$ is the rotation angle of $i$ th quantum bit, and $\theta_{i}=e^{d / d^{\prime}} \times \Delta \theta \times S\left(\alpha_{j i}, \beta_{j i}\right)$. Where $\Delta \theta$ and $S\left(\alpha_{j i}, \beta_{j i}\right)$ represent the size and direction of $\theta_{i}$, respectively, can be found in a table [32], $d$ is the distance between AUV and obstacles, hybrid QACO will be called when $d \leq d^{\prime}$. In the early stage of the algorithm, $\theta_{i}$ is relatively large, which can improve the search efficiency of hybrid QACO. And with the increase of the number of iterations, $\theta_{i}$ decreases gradually, which improves the local search performance. 


\subsection{Translocation Strategy}

Suppose ant $j$ at path point $a$ in current time, then the next path node $b$ of ant $j$ can be select as follows:

$$
b=\left\{\begin{array}{cl}
\underset{s \in \mathbf{S}}{\arg \max }\left\{[\tau(a, s)]^{\xi} \cdot[\eta(a, s)]^{\gamma}\right\}, & q \leq q_{0} \\
b^{\prime}, & \text { otherwise }
\end{array}\right.
$$

where $\tau(a, b)$ represent the pheromones of path $(a, b)$ determined by $\alpha, \beta \cdot \eta(a, b)=\left(1 / d_{a b}\right)$ is heuristic information of $(a, b), \xi$ and $\gamma$ are the weights of $\tau(a, b)$ and $\eta(a, b)$. $\mathbf{S}$ is the points set held by ant colony, $b^{\prime}$ is the next point selected according to (12):

$$
p(a, b)=\left\{\begin{array}{cl}
\frac{\tau(a, b)^{\xi} \cdot \tau(a, b)^{\gamma}}{\sum_{s \in S} \tau(a, s)^{\xi} \cdot \tau(a, b)^{\gamma}}, & b \in \mathbf{S} \\
0, & \text { otherwise }
\end{array}\right.
$$

\subsection{Hybrid QACO Pheromone Updating Rule}

\subsubsection{Local Update for Pheromone}

In this paper, a nonlinear function $n(\theta)$ is defined to limit the turn angle of AUV:

$$
\begin{array}{ll}
\text { if } \quad 0^{\circ} \leq|\theta| \leq 15^{\circ} & \text { then } n(\theta)=0 \\
\text { if } 15^{\circ}<|\theta| \leq 30^{\circ} & \text { then } n(\theta)=20 \\
\text { if } 30^{\circ}<|\theta| \leq 45^{\circ} & \text { then } n(\theta)=40 \\
\text { if } 45^{\circ}<|\theta| \leq 60^{\circ} & \text { then } n(\theta)=80
\end{array}
$$

A nonlinear punishment mechanism is introduced in the objective function to avoid AUV turn angle overlarge. The fitness function of path $f_{j}$ can expressed as follows:

$$
\begin{gathered}
f_{j}=\varepsilon_{1} \text { sum }_{j}+\varepsilon_{2} \text { dist }_{j}+\varepsilon_{3} f a_{j} \\
f a_{j}=-\left(n\left(\theta 1_{i, i+1}\right)+n\left(\left|\theta 2_{i, i+1}\right|+10\right)\right) \cdot \sum_{i=0}^{n-1} v
\end{gathered}
$$

where dist $_{j}$ is the Euclidean distance between the next point obtained by ant $j$ and the goal point, $s m_{j}$ is the distance of a tour built by ant $j, f a_{j}$ represent the yaw fitness of the path built by ant $j, v$ is a positive number close to zero, $\theta 1$ and $\theta 2$ are the yaw angle and pitch angle of AUV, respectively, and $\theta 2$ is set to zero when AUV navigating in a two-dimensional environment.

Then the local updating of the pheromone as follows:

$$
\begin{aligned}
& \tau_{j i}(t+1)=(1-\rho) \tau_{j i}(t)+\rho \sum_{k=1}^{n} \Delta \tau_{j i}^{k} \\
& \Delta \tau_{j i}^{k}=\left\{\begin{array}{cl}
Q \cdot f_{j}, & \text { if } \text { ant }_{j} \text { passed } e_{j i} \\
0, & \text { otherwise }
\end{array}\right.
\end{aligned}
$$

where $\rho$ is the evaporation rate, $Q$ is a parameter that is relative to the speed of convergence, $f_{j}$ is the fitness function of the path built by ant $j$.

\subsubsection{Global Update for Pheromone}

Step 1: Finding out the best ant ant ${ }_{o p(t)}$ at each generation. Then do

$$
\begin{gathered}
\tau_{j i}(t+1)=\varphi \tau_{j i}(t) \\
\varphi= \begin{cases}k * f_{o p(t)}, & k * f_{o p(t)}>1 \text { and } \operatorname{ant}_{o p(t)} \text { passed } e_{j i} \\
C_{1}, & k * f_{o p(t)} \leq 1 \text { and } \operatorname{ant}_{o p(t)} \text { passed } e_{j i} \\
0, & \text { otherwise }\end{cases}
\end{gathered}
$$

where $\varphi$ is the magnifying multiple, $\mathrm{C}_{1}\left(\mathrm{C}_{1}>1\right)$ is a constant.

Step 2: $L_{o p(t)}$ is the shortest distance at each generation. ant $_{o p(t)}$ is the ant that found out the best path at the last generation. If $L_{o p(t)}>L_{o p(t-1)}$, there is no need to take this global updating. Otherwise, the pheromone is updated according to the following functions:

$$
\psi= \begin{cases}\tau_{j i}(t+1)=\psi \tau_{j i}(t) \\ C_{o p(t)}, & k * f_{o p(t)}>1 \text { and } \\ \text { ant }_{o p(t)} \text { passed } e_{j i} \text { while ant }_{o p(t-1)} \text { didn't } & k * f_{o p(t)} \leq 1 \text { and } \\ & \text { ant }_{o p(t)} \text { passed } e_{j i} \text { while ant }_{o p(t-1)} \text { didn't } \\ 0, & \text { otherwise }\end{cases}
$$

where $\psi$ is the magnifying multiple, $C_{2}\left(C_{2}>1\right)$ is a constant.

\subsubsection{The Limited of Pheromone}

The pheromone should be limited according to MMAS:

$$
\tau_{j i}(t+1)= \begin{cases}\tau_{\min }, & \text { if } \tau_{i j}(t+1)<\tau_{\min } \\ \tau_{\max }, & \text { if } \tau_{i j}(t+1)<\tau_{\max } \\ \tau_{j i}(t+1), & \text { otherwise }\end{cases}
$$

\subsubsection{A Local Search Method for AUV Path Plan}

To improve the quality of the path planned by QACO and obtain a smoother path, a local search method is proposed in this paper. This local search method is applied to each new position found by each ant during the search process. 
Start from the initial position, the local search method is used to determine the feasibility of each new position. If the new position is feasible, then the local search method is used to find a batter. Else the method is used to find a feasible position.

\subsubsection{The Step of Online Path Planning Based on Pheromone}

Step 1: Initialize all parameters of hybrid QACO. $n$ is the total number of ants in the ant colony; $m$ is the dimension of quantum bit; $k$ is the number of path node in each dimension. All $\alpha$ and $\beta$ in $P$ are initialized to $1 / \sqrt{2}$.

Step 2: Put all ants on the current position of AUV.

Step 3: Ants choose next nodes by a probabilistic state transition rule given by (11), (12), and build

$$
\begin{aligned}
& \mathbf{P}(t)=\left\{\mathbf{p}_{1}^{t}, \mathbf{p}_{2}^{t}, \ldots, \mathbf{p}_{n}^{t}\right\}, \text { where } \\
& \mathbf{p}_{j}^{t}=\left[\begin{array}{c|c|c|c}
\alpha_{j 1}^{t} & \alpha_{j 2}^{t} & \ldots & \alpha_{j m}^{t} \\
\beta_{j 1}^{t} & \beta_{j 2}^{t} & \ldots & \beta_{j m}^{t}
\end{array}\right] .
\end{aligned}
$$

Step 4: Determine whether each note in $\mathbf{P}(t)$ is feasible, then use local search method to optimize or amend $\mathbf{P}(t)$.

Step 5: Building feasible path $\mathbf{R}(t)=\left\{r_{1}^{t}, r_{2}^{t}, \ldots, r_{n}^{t}\right\}$ for each ant by observing $P(t)$. Specifically

$$
\begin{aligned}
& \text { If } \sigma \leq \sigma_{0} \\
& r_{j, i}= \begin{cases}\text { node } 0, & \beta_{j, i} \leq \alpha_{j, i} \\
\text { node } 1, & \beta_{j, i}>\alpha_{j, i}\end{cases}
\end{aligned}
$$

Else

$$
r_{j, i}= \begin{cases}\text { node } 0, & \mu \leq \mu_{0} \\ \text { node } 1, & \mu>\mu_{0}\end{cases}
$$

where $r_{j, i}$ represent the state of ant $j$ in the $i$ th dimension, the phenomenon of node 0 and node 1 are $\left|\alpha_{j, i}\right|^{2}$ and $\left|\beta_{j, i}\right|^{2}$, respectively. $\sigma, \mu \in(0,1)$ are random numbers, $\sigma_{0}, \mu_{0} \in(0,1)$ are constants.

Step 6: Update local pheromone according to (16) and (17). If all the ants complete traversal, jump to Step 7, else return to Step 3.

Step 7: Calculate fitness function of each ant by (14). And choose the optimal ant in each generation.

Step 8: Determine whether the termination condition is satisfied, if true, output the optimal path.

Step 9: Update global phenomenon according to (18)-(21).

Step 10: Update phenomenon by quantum rotation gate.

Step 11: Update the information of environment and $t=t+1$.

\section{Online Path Planning Based on Hybrid QPSO}

\subsection{Environmental Model and Description of Optimization Problem}

When AUV navigate in an unknown environment, due to the limited ability of the detector to perceive the obstacle,

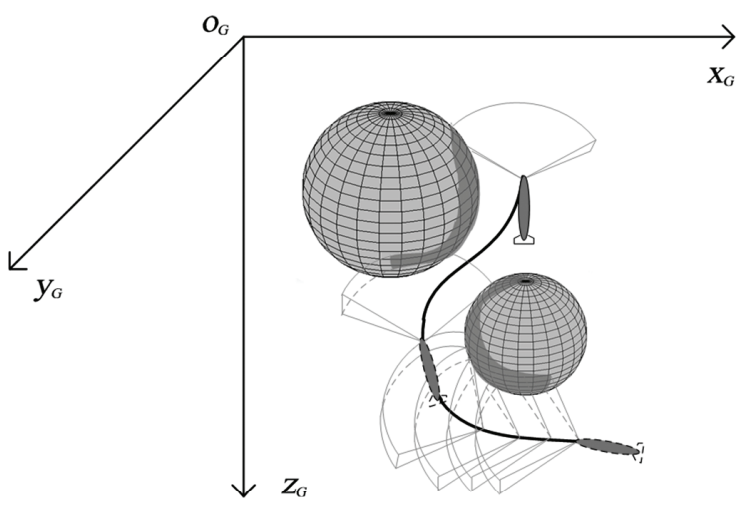

Figure 3. The detecting procedure of sonar.

the environment should be modelled locally.

In this paper, a method of rolling optimization window is used to online plan path for AUV. Considering the perceived range of the sensor, the operating environment is divided into a series of moving windows. And the window moves forward with AUV. When the hybrid QACO is called, it will plan a shortest and collision-free path from the current location of AUV to the next path point of goal path according to the current environment information. The next path nodes are selected in a shell area which is divided into $k \times h \times m$ parts. The horizontal contained angle and vertical contained angle are set to $120^{\circ}$ and $17^{\circ}$, respectively, and the radius of the sell area is set to $100 \mathrm{~m}$. For two-dimensional environment, the path nodes are selected in a sector area. The detecting procedure of multibeam FLS is shown in Fig. 3. In which, the bold line is the track of AUV, and the known environment information is highlighted.

\subsection{The Processes of Online Path Planning}

Step 1: Initialize relevant parameters, such as the starting position, the target position and so on.

Step 2: Plan a global path from start point to target point. Step 3: AUV navigates along the line from the current point to the target point, and then the information of map is updated in real time based on the information from multibeam FLS.

Step 4: According to the information of map to determine whether to reach the target point, and if so, the algorithm is stopped.

Step 5: If the conflict exits between obstacles have detected by FLS and global path of AUV, and the distance of surface of obstacles and AUV is less than a fixed value, then the hybrid QACO is called to avoid the obstacles. Step 6: When AUV avoids obstacles totally, return to the global path, then jump to Step 3 .

\section{Simulations}

\subsection{Simulation Results in Two-Dimensional Envi- ronment}

Suppose AUV navigates in the area of 1,000 $\mathrm{m} \times 1,000 \mathrm{~m}$, its rate is limited in $2-4 \mathrm{kn}$. The detection frequency and 


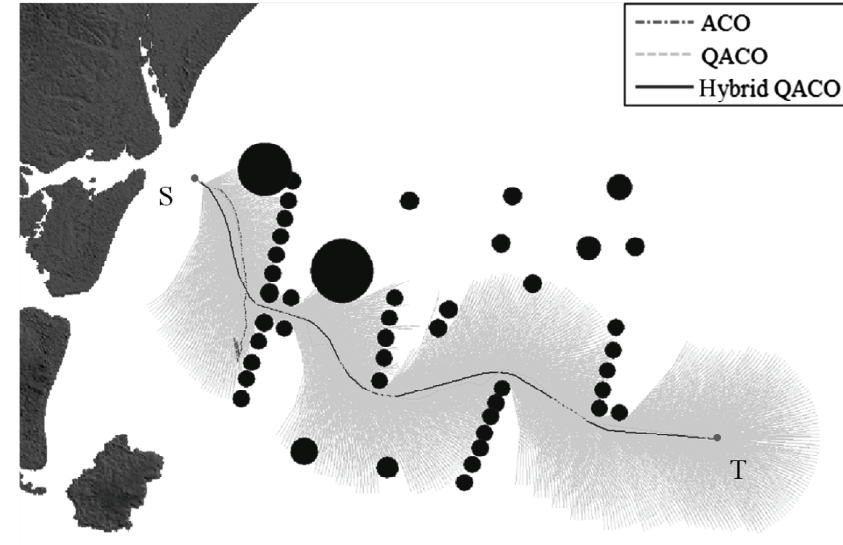

(a)

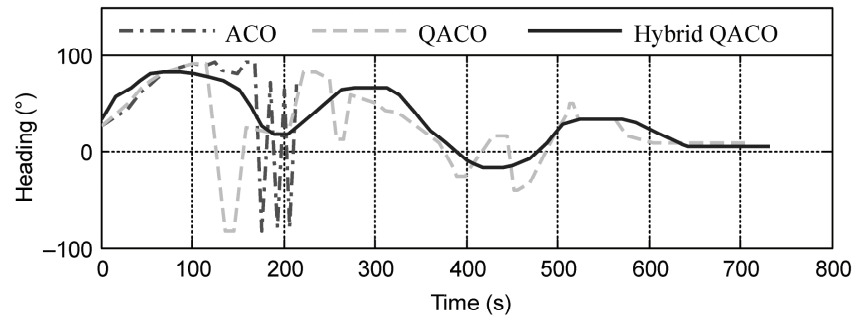

(b)

Figure 4. Tracks (a) and headings (b) of AUV planned by ACO, QACO and hybrid QACO in environment 1.

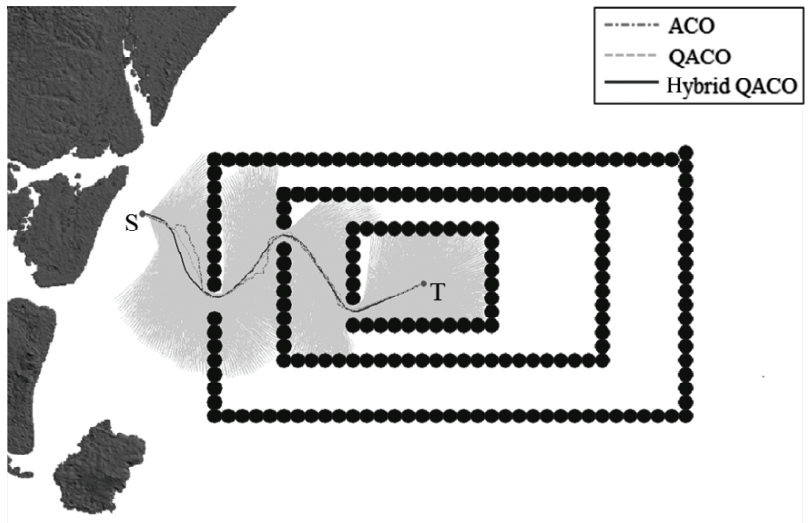

(a)

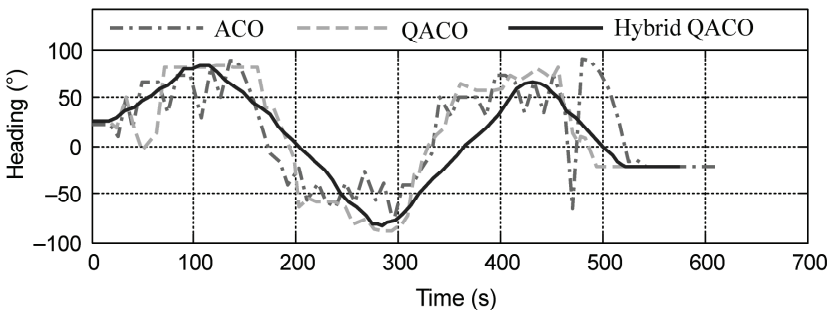

(c)

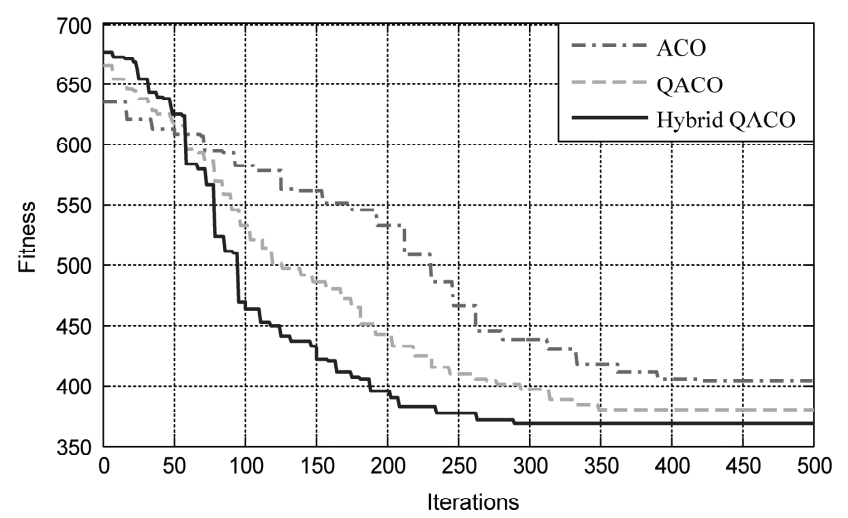

(b)

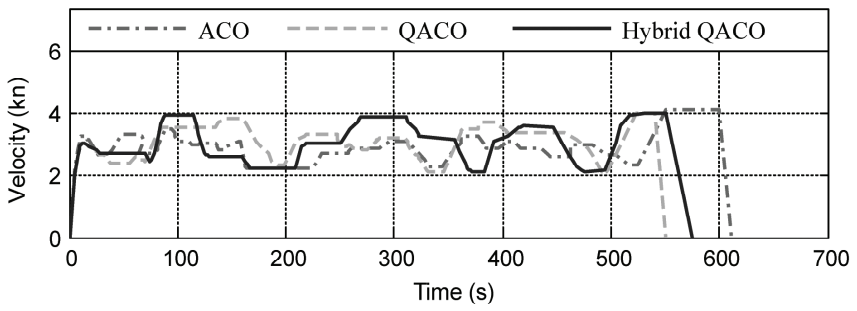

(d)

Figure 5. Tracks (a), fitness (b), headings (c) and velocity (d) of AUV planned by ACO, QACO and QACO in environment 2.

distance of simulation sonar is $5 \mathrm{~Hz}$ and $120 \mathrm{~m}$. When the distance between AUV and obstacles is less than $80 \mathrm{~m}$, call the algorithm for path planning. Let $n=40$, $q_{0}=0.2$, the safety distance $r=7 \mathrm{~m}$, the maximum generation $\operatorname{Max}=500$, the dimension $k=80, m=2$.

To evaluate the contribution of local search method in $\mathrm{QACO}$, the performance of ACO QACO and hybrid QACO is compared, respectively, in the following simulation cases. Figure 4 shows the simulation results of the three path planning algorithms in environment $1 . \mathrm{ACO}$ is failing to find the feasible path point in the narrow pass way. Figure 4 indicates that the quality and smoothness of the path are improved through the combination of hybrid QACO and
QACO. And the path obtained by the hybrid QACO is more suitable for the dynamic characteristics of AUV.

Figure 5 shows the simulation results of ACO, QACO and hybrid QACO in environment 2. Three paths are shown in Fig. 5(a). Figure 5(b) shows the yaw angle of AUV. This result shows that the hybrid QACO is more suitable for the yaw characteristics than ACO and QACO. Figure 5(c) shows the velocity of AUV on the three paths. Figure 5(d) compares the mean path fitness obtained by ACO, QACO and hybrid QACO over 20 simulations. The comparison of fitness curves clearly shows that the hybrid QACO has stronger searchability compared with ACO and QACO. To compare the robustness of the two algorithms, 
Table 1

Path Length Comparison of Hybrid QACO, QACO and ACO

\begin{tabular}{|l|c|c|c|}
\hline Algorithms & $\begin{array}{c}\text { Optimal Length } \\
(\mathrm{m})\end{array}$ & $\begin{array}{c}\text { Average Path } \\
\text { Length }(\mathrm{m})\end{array}$ & $\begin{array}{c}\text { Average Computation } \\
\text { Time }(\mathrm{ms})\end{array}$ \\
\hline ACO & 611.84 & 637.73 & 267 \\
\hline QACO & 585.23 & 597.72 & 294 \\
\hline Hybrid QACO & 583.64 & 588.35 & 308 \\
\hline
\end{tabular}

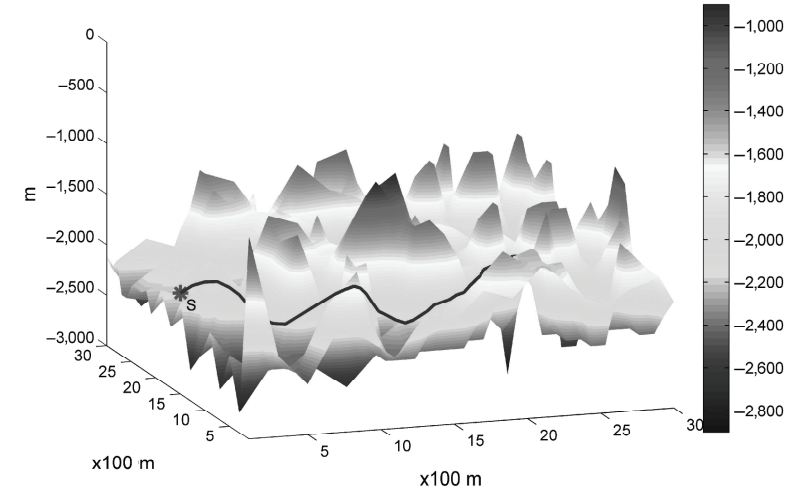

(a)

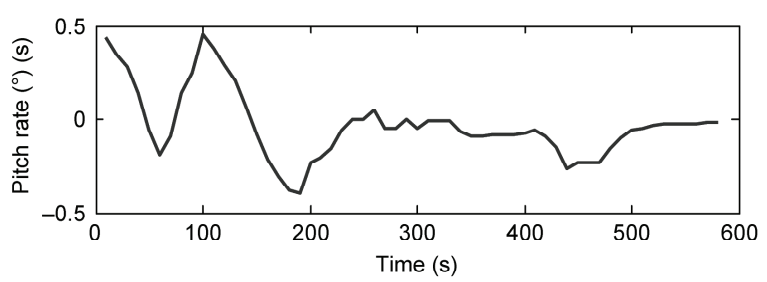

(c)

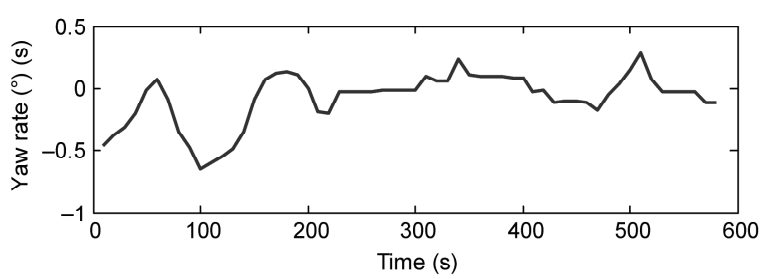

(b)

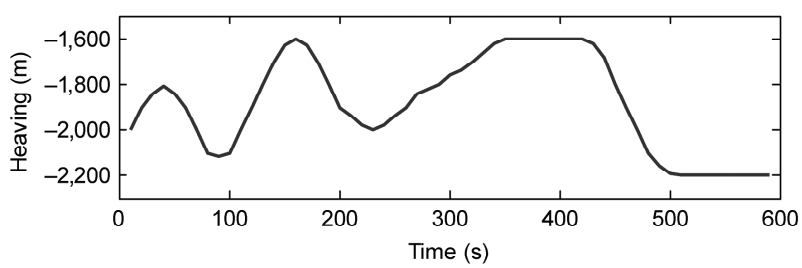

(d)

Figure 6. The track (a), yaw rate(b), pitch rate (c) and heaving(d) of AUV planned by hybrid QACO in three-dimensional environment.

the average value and optimal value based on 30 runs of ACO, QACO and hybrid QACO are shown in Table 1. Table 1 confirms that hybrid QACO has better robustness than ACO and QACO. The average computation time of the three algorithms is also shown in Table 1. Compared to QACO and ACO, the hybrid QACO only increases a little computation time, but the performance of path planning is improved significantly both in optimizing ability and in smoothness of path.

\subsection{Simulation Results Based on Hybrid QACO in Three-Dimensional Environment}

In a three-dimensional environment, AUV navigates in the area of $3,000 \mathrm{~m} \times 3,000 \mathrm{~m} \times 2,000 \mathrm{~m}$, and the velocity of AUV is set to $5 \mathrm{kn}$. The detection frequency and distance of simulation sonar are $5 \mathrm{~Hz}$ and $120 \mathrm{~m}$. When the distance between AUV and obstacles is less than $100 \mathrm{~m}$, call the algorithm for path planning. Let $n=40, q_{0}=0.2$, the safety distance $r=7 \mathrm{~m}$, the maximum generation $\operatorname{Max}=500$, the dimension of environment model $k=80, h=17, m=2$. The start point and target point are set to $(0,1,500,1,000)$ and $(3,000,600,800)$, respectively. The simulation results are shown in Fig. 6. The path of AUV is shown in Fig. 6(a). AUV always navigates towards the target until obstacles are detected by sonar. Figure 6(b) and 6(c) shows the yaw rate and pitch rate of AUV, respectively. The results show that the path planned by hybrid QACO is suitable for the yaw and pitch characteristics of AUV. And the heaving of AUV is shown in Fig. 6(d).

\section{Conclusion}

This paper proposed a method of online path planning for AUV based on hybrid QACO. A rolling optimization window and a new local environment model are built in the detection range of multibeam FLS. In the hybrid QACO, an adaptive quantum gate is defined for online path planning. A fitness function is proposed to avoid the appearance of large yaw. According to the actual situation, the pheromone is updated globally and the pheromone 
on the best path is strengthened solely. A local search method is combined with QACO to improve the quality of the path planned by QACO and obtain a smoother path. The simulation experiment is carried out to test the performance of the algorithm. The simulation results show the good performance of this algorithm.

\section{Acknowledgement}

This research work is supported by the China Natural Science Foundation (Grant No. 61633008) and the Natural Science Foundation of Heilongjiang Province (Grant No. F2015035).

\section{References}

[1] X. Wang, M. Zhou, X. Liu, and Y. Fan, The research on robot global path planning based on simulated annealing dissipative ant system, World Congress on Intelligent Control and Automation, Chongqing, China, 2008, 5764-5769.

[2] K. H. Sedighi, K. Ashenayi, T. W. Manikas, and R. L. Wainwright, Autonomous local path planning for a mobile robot using a genetic algorithm, Congress on Evolutionary Computation, Portland, OR, USA, 2004, 1338-1345.

[3] G. Zhang and H. Jia, 3D path planning of AUV based on improved ant colony optimization, Control Conference, Xi'an, China, 2013, 5017-5022.

[4] J. Liu, J. Yang, H. Liu, and X. Tian, An improved ant colony algorithm for robot path planning, Soft Computing, 1(11), 2016, 1-11.

[5] Y. Yao, Q. Ni, Q. Lv, and K. Huang, A novel heterogeneous feature ant colony optimization and its application on robot path planning, IEEE Congress on Evolutionary Computation, Sendai, Japan, 2015, 522-528.

[6] J. Zhao, D. Cheng, and C. Hao, An improved ant colony algorithm for solving the path planning problem of the omnidirectional mobile vehicle, Mathematical Problems in Engineering, 2016, 2016, 1-10.

[7] U. Cekmez, M. Ozsiginan, and O. K. Sahingoz, Multi colony ant optimization for UAV path planning with obstacle avoidance, International Conference on Unmanned Aircraft Systems, 2016, $47-52$.

[8] J. Cao, Robot global path planning based on an improved ant colony algorithm, Journal of Computer and Communications, 4(2), 2016, 11-19.

[9] L. Deng, X. Ma, G. Jason, Y. Li, Z. Xu, and Y. Wang, Artificial immune network-based multi-robot formation path planning with obstacle avoidance, International Journal of Robotics and Automation, 31(3), 2016.

[10] J. Li, G. Deng, C. Luo, et al., A hybrid path planning method in unmanned air/ground vehicle (UAV/UGV) cooperative systems, IEEE Transactions on Vehicular Technology, 65(12), 2016, 9585-9596.

[11] D. Fan and P. Shi, Improvement of Dijkstra's algorithm and its application in route planning, Int. Conf. on Fuzzy Systems 8 Knowledge Discovery IEEE, Yantai, China, 2010, 1901-1904.

[12] D. Dolgov, S. Thrun, M. Montemerlo, et al., Path planning for autonomous driving in unknown environments, Springer Tracts in Advanced Robotics, 54, 2009, 55-64.

[13] J. Nieto, E. Slawinski, V. Mut, et al., Online path planning based on rapidly-exploring random trees, IEEE Int. Conf. Industrial Technology, Vina del Mar, Chile, 2010, 1451-1456.

[14] Y. Zhang, W. Li, and C. Silva. RSMDP-based robust Qlearning for optimal path planning in a dynamic environment, International Journal of Robotics and Automation, 31(4), 2016, 290-300.

[15] A. Zhu, Y. Chen, and C. Luo, A machine learning based algorithm for detecting a moving object, International Journal of Robotics 83 Automation, 31(5), 2016, 402-408.

[16] X. Chen, G. Tan, and B. Jiang, Real-time optimal path planning for mobile robots based on immune genetic algorithm, Journal of Central South University, 39(3), 2008, 577-583.
[17] J. Yang, Y. Chen, C. Fan, and P. Tseng, Real-time path planning for unmanned surface vehicle by using finite angle A* algorithm, Journal of Taiwan Society of Naval Architects and Marine Engineers, 2015, 34(3), 165-172.

[18] H. Yu, A. Shen, and Y. Su, Continuous motion planning in complex and dynamic underwater environments, International Journal of Robotics and Automation, 30(2), 2015, 192-204.

[19] D. Zhu, C. Cheng, and B. Sun, An integrated AUV path planning algorithm with ocean current and dynamic obstacles, International Journal of Robotics and Automation, 31(5), 2016, 382-389.

[20] D. Gong, L. Lu, and M. Li, Robot path planning in uncertain environments based on particle swarm optimization, Eleventh Conf. on Congress on Evolutionary Computation, Trondheim, Norway, 29(5), 2009, 2127-2134.

[21] Z. Cheng, E. Wang, Y. Tang, and Y. Wang, Real-time path planning strategy for UAV based on improved particle swarm optimization, Journal of Computers, 9(1), 2014, 209-214.

[22] T. Huang, P. Yang, and K. Yang, Navigation of mobile robot in unknown environment based on T-S neuro-fuzzy system, International Journal of Robotics and Automation, 30(4), 2015, 384-396.

[23] J. Sfeir, M. Saad, and H. Saliah-Hassane, An improved artificial potential field approach to real-time mobile robot path planning in an unknown environment, IEEE Int. Symp. on Robotic \& Sensors Environments, Montreal, QC, Canada, 2011, 208-213.

[24] B. Zeng and Y. Yang, Method of real-time path planning based on ant colony algorithm in dynamic environment, Application Research of Computers, 27(3), 2010, 860-863.

[25] C. Hsu, R. Hou, and W. Wang, Path planning for mobile robots based on improved ant colony optimization, IEEE Int. Conf. on Systems, Manchester, UK, 2013, 2777-2782.

[26] L. Wang, Q. Niu, and M. Fei, A novel quantum ant colony optimization algorithm, Bio-Inspired Computational Intelligence and Applications. Berlin, Germany: Springer-Verlag, 2007, 277-286.

[27] G. Yue, J. Zhao, and Y. Zhang, Improved quantum ant colony algorithm for TSP, Computer Engineering 83 Design, 30(16), 2009, 3843-3832.

[28] C. Pan, Y. Yang, and Q. Zhang, Phase encoded-based quantum ant colony algorithm with application to continuous optimization, Application Research of Computers, 27(12), 2010, 4450-4453.

[29] Y. Ma, W. Tian, and Y. Fan, Improved quantum ant colony algorithm for solving TSP problem, 2014 IEEE Workshop on Electronics, Computer and Applications, Ottawa, ON, Canada, 2014, 453-456.

[30] H. Liu, X. Bian, J. Li, and H. Wang, Study of local path planning based on forward looking sonar for AUV, Microcomputer Information, 23(23), 2007, 243-245.

[31] Q. Chen, Research on autonomous planning method of multiple unmanned underwater vehicles cooperative executing multiple task, Master's Degree Thesis, Harbin Engineering University, 2015.

[32] J. Zhang, A research on modeling and simplifying of the moving submarine, Master's Degree Thesis, Harbin Engineering University, 2009

\section{Biographies}

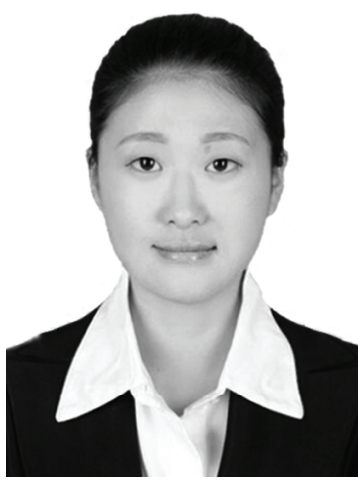

Changjian Lin is studying for a doctor's degree at Harbin Engineering University. She received her Bachelor's degree in Changchun University of Science and Technology. Her researches focus on intelligent control method used in unmanned systems. 


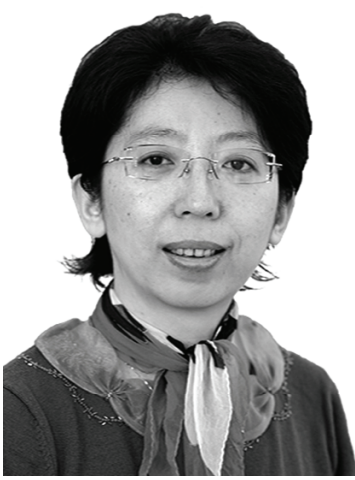

Hongjian Wang completed her Ph.D. degree from Harbin Engineering University in 2004. She works mainly in the area of autonomous underwater vehicle control, robotic system and intelligent control, shipboard engineering dynamic positioning and so on.

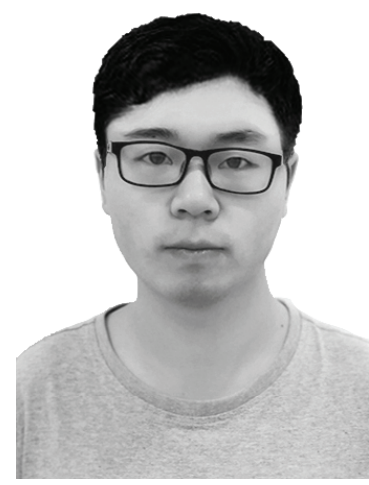

Jianya Yuan has received his Bachelor's degree in Automation from Mudanjiang Normal University. He is studying for a doctor's degree at Harbin Engineering University. His current research interests include multi-agent, path planning, and Deep Q-learning.

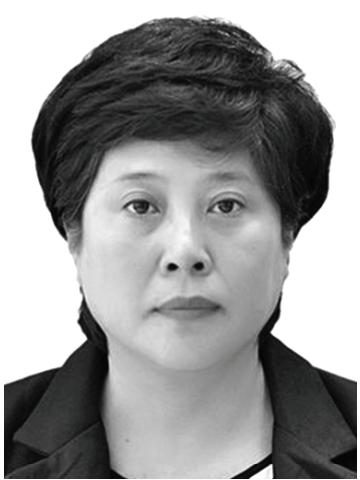

Mingyu $F u$ has obtained her Ph.D. degree in 2005 from Harbin Engineering University. She leads multiple many research projects and makes many remarkable achievements in ship dynamic positioning, hovercraft integrated driving control, ship motion control and other fields. 